\title{
Computational Aeroacoustics beneath High Speed Transitional and Turbulent Boundary Layers
}

\author{
Konstantinos Ritos $^{a, 1}$, Dimitris Drikakis ${ }^{b, *, 2}$, Ioannis W. Kokkinakis ${ }^{a, 3}$ and S. \\ Michael Spottswood ${ }^{c, 4}$ \\ ${ }^{a}$ Department of Mechanical \& Aerospace Engineering, University of Strathclyde, Glasgow, G1 1XJ, UK \\ ${ }^{b}$ Defence and Security Research Institute, University of Nicosia, Nicosia, Cyprus \\ ${ }^{c}$ Air Force Research Laboratory, Wright Patterson AFB, Ohio 45433-7402, USA
}

\section{ARTICLE INFO}

Keywords:

Aeroacoustics

Hypersonic flow

Transition

Turbulence

Compressible flow

Direct Numerical Simulation

Shock-boundary layer interaction.

\begin{abstract}
A B S TR ACT
This paper concerns a study of pressure fluctuations beneath hypersonic shock-wave turbulent boundary layer interactions and the associated acoustic loading on a compression/expansion ramp. Using high-order methods, we have performed Direct Numerical Simulations at Mach 7.2. We compare the spectral analysis of the pressure fluctuations at various locations of the compression/expansion ramp with the spectra calculated beneath a hypersonic transitional boundary layer. Similarities and differences between the two hypersonic boundary layers, in the context of acoustic loading, are drawn. Extremely high values of pressure fluctuations are recorded after the shock re-attachment where we also observe the maximum pressure gradients indicating that acoustic loading is correlated with areas of high-pressure gradients. Finally, we discuss the impact of the boundary layer state (attached flow, turbulence bursts, recirculations, shock oscillations, shock re-attachment and expansion fans) on the frequency spectrum of the pressure fluctuations.
\end{abstract}

\section{Introduction}

Structural elements of supersonic and hypersonic aircraft are exposed to acoustic fatigue due to pressure fluctuations beneath the transitional or turbulent boundary layers (TBLs), as well as to shock-wave boundary layer interaction (SBLI). There is a body of literature concerning subsonic and supersonic TBLs, including publications concerning the amplitude of pressure fluctuations [1] and the roll-off of the pressure spectrum [2, 3, 4]. These models and theories have been validated experimentally $[5,6,7,8]$ and also verified numerically $[9,10]$.

To date, the most relevant and comprehensive study on the response of a thin, flexible panel to SBLI is the experimental campaign conducted by AFRL Structural Sciences Center at Mach 2 [11], where they have investigated both the amplitude of pressure fluctuations and roll-off of pressure. Furthermore, an overview of experiment and challenges relevant to aerothermoelastic testing in high-temperature, high-speed wind tunnels was also published [12]. In [11],

*Corresponding author

drikakis.d@unic.ac.cy (D. Drikakis)

$\operatorname{ORCID}(\mathrm{s})$ :

${ }^{1}$ Lecturer, Mechanical \& Aerospace Engineering, University of Strathclyde, Glasgow, UK.

${ }^{2}$ Professor and Executive Director of Research and Innovation, University of Nicosia, Cyprus; also Senior Researcher at the University of Nicosia Research Foundation

${ }^{3}$ Lecturer, Mechanical \& Aerospace Engineering, University of Strathclyde, Glasgow, UK.

${ }^{4}$ Principal Aerospace Engineer, Air Force Research Laboratory, Wright Patterson AFB, Ohio 45433-7402, USA. 
the authors observed and measured the effect of turbulence, SBLI and heated flow on an aircraft-like panel, and explored severe structural events such as dynamic instabilities and material failure. Furthermore, they refine full-field and non-contacting experimental measurement techniques necessary to characterize the flow environment and structural response. The above experimental studies, together with finite element models for predicting the nonlinear response of an aerospace structure [13], can significantly advance the design of thin structures such as beams, panels, and shells.

Concerning hypersonic transitional and turbulent boundary layers, with or without SBLI, and their effects on acoustic loading, there are still significant gaps of knowledge. Past numerical [14, 15] and experimental [16, 17, 18, 19] studies of hypersonic SBLI have focused on the structure of turbulence. For instance, Görtler-like vortices have been observed at Mach 7 around a compression ramp, and studies are suggesting these vortices are responsible for the lowfrequency motion of the shock wave [15]. Studies of hypersonic TBLs have also been published [20, 21]; however investigations of the effects of transitional or turbulent boundary layers on acoustic loading are scarce [22, 23, 24, 25].

In this paper, we present a spectral analysis of pressure fluctuations beneath hypersonic SBLI by performing DNS of a Mach 7.2 turbulent boundary layer over a $33^{\circ}$ compression ramp. We have chosen the ramp angle, and free-stream properties according to the experimental set up of Schreyer et al. [19]. One or more shocks form in the supersonic and hypersonic parts of the flow and the interaction of the shock system with the incoming turbulent boundary layer leads to significant pressure gradients. We discuss how the above system influences the frequency spectrum at the end of the transition and in the fully turbulent region.

\section{Governing equations and numerical modeling}

We have employed DNS in the framework of the block-structured code CNS3D [26, 27, 28]. The code solves the full Navier-Stokes equations using a finite volume Godunov-type method for the convective terms. We discretise the inter-cell numerical fluxes by solving the Riemann problem using the reconstructed values of the primitive variables at the cell interfaces. We use a one-dimensional swept unidirectional stencil for the reconstruction of the variables. The Riemann problem is solved using the so-called "Harten, Lax, van Leer, and (the missing) Contact" (HLLC) approximate Riemann solver [29, 30]. We have implemented the 9th-order Weighted-Essentially-Non-Oscillatory (WENO) scheme [31] in conjunction with the HLLC solver. We use a second-order central scheme for the discretisation of the viscous terms are discretised using. The solution is advanced in time using a five-stage (fourth-order accurate) optimal strong-stability-preserving Runge-Kutta method [32].

\subsection{Hypersonic flow over a flat plate}

Two flow cases are considered here, with the first one being a hypersonic flow over a flat plate subjected to von Kármán atmospheric spectrum at the inlet. The flow transitions to fully turbulent at a downstream location as shown in 


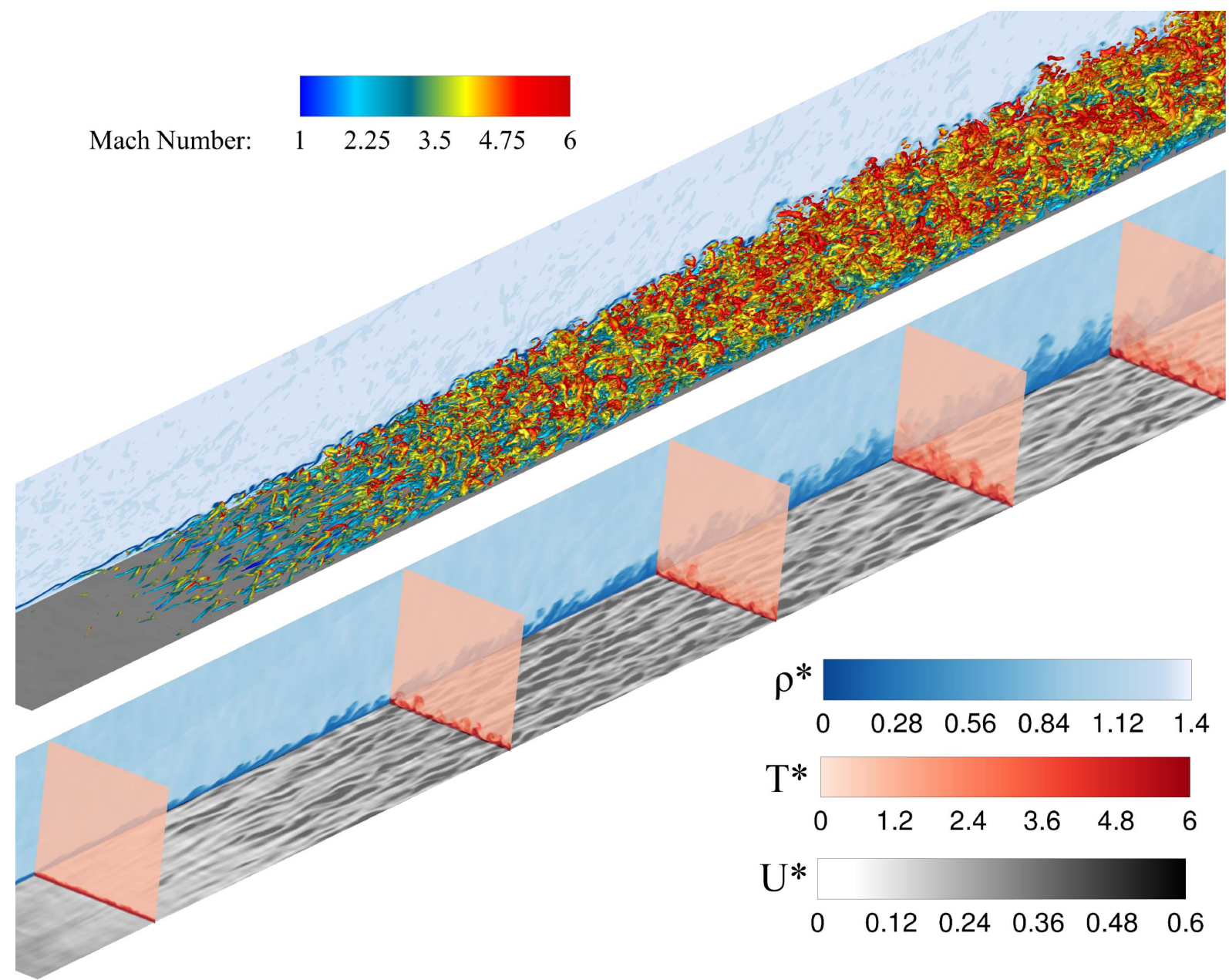

Figure 1: Isosurfaces of compressible Q-criterion coloured by Mach number. The density gradient is also plotted in grayscale on the side of the graph highlighting the transition region. Contour plots of density and streamwise velocity near the wall, as well as temperature contour plots at various cross sections of the simulation domain, are also shown.

Figure 1 by isosurfaces of compressible Q-criterion and contour plots of density and temperature. The results presented here are from simulations performed at Mach 6 and turbulence intensity of the free-stream velocity $T u=3 \%$. Further details about the free-stream and boundary conditions can be found in [25].

To examine mesh resolution effects, a study was performed using three meshes, $G 1=661 \times 161 \times 91, G 2=$ $811 \times 201 \times 121$ and $G 3=1001 \times 251 \times 151$. All meshes are relatively fine and qualitatively comparable to previous DNS simulations of fully-turbulent hypersonic flows [33, 22].The different mesh sizes similarly predict the transition point and the shape factor $H$ (Figure 2). The shape factor $H$ is used to assess the compressible boundary layer flow; $H$ is defined as the ratio of the displacement thickness $\left(\delta^{*}\right)$ to the momentum thickness $(\theta)$ : 


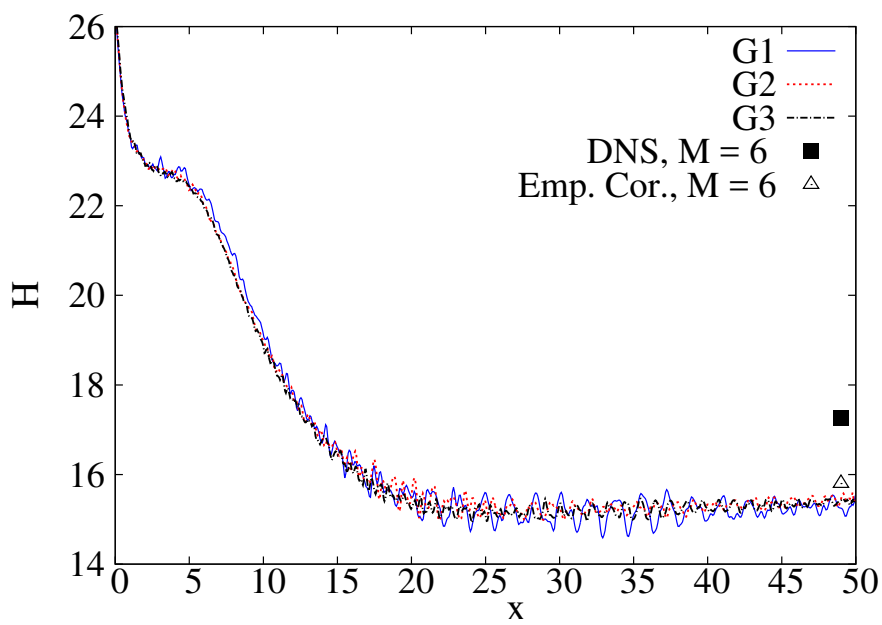

Figure 2: Shape factor $H$ development along the plate for three mesh resolutions. An empirical correlation (Eq. 2) and DNS data are also presented for the fully turbulent flow regime.

$$
H=\frac{\delta^{*}}{\theta}=\left[\int_{0}^{\infty}\left(1-\frac{\rho(y) u(y)}{\rho_{\infty} u_{\infty}}\right) d y\right]\left[\int_{0}^{\infty} \frac{\rho(y) u(y)}{\rho_{\infty} u_{\infty}}\left(1-\frac{u(y)}{u_{\infty}}\right) d y\right]^{-1},
$$

where $u_{\infty}$ and $\rho_{\infty}$ are the freestream velocity and density, respectively. Previous studies [34, 35, 36, 37] have confirmed the reduction of the shape factor with increasing Reynolds number and the potential asymptotic limit at high Reynolds numbers. A correlation formula for fully turbulent boundary layers and a broad range of Reynolds numbers has been proposed in the literature [37]

$$
H_{c o m p}=H_{i n c}+0.4 M^{2}+1.222 \frac{T_{w}-T_{a w}}{T_{\infty}}
$$

where $H_{\text {inc }}=1.4$. The present simulation results are in good agreement with the above formula's predictions (Figure 2). The DNS data in Figure 2 are from simulations with different flow conditions and Reynolds number than our simulations, and this explains the discrepancies in the shape factor values. Flow statistics are computed by averaging in time over three flow cycles and spatially in the spanwise direction. The total simulation time is equal to six flow cycles.

\subsection{Hypersonic flow over a compression/expansion ramp}

The second case considered here is a hypersonic flow over a $\alpha=33^{\circ}$ compression ramp followed by an expansion corner with the same deflection angle (Figure 3). The incoming flow corresponds to a turbulent boundary layer at Mach 7.2 with a thickness $\delta=5 \mathrm{~mm}$. Based on the free-stream properties (Table 1) and the reference length $\delta$, the incoming flow has a Reynolds number of $R e_{\delta}=102,731$. We implement periodic boundary conditions in the spanwise direction 


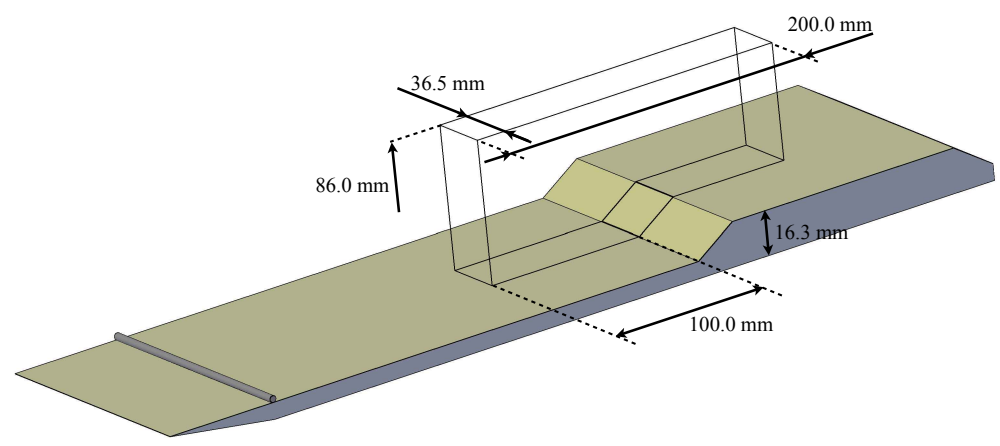

Figure 3: Schematic representation of the simulation domain with dimensions in $\mathrm{mm}$.

(z). In the wall-normal direction (y), we use a no-slip isothermal wall (with a temperature $\mathrm{T}_{w}$ of $340 \mathrm{~K}$ ) [38]. Highorder implementation of the boundary conditions requires fictitious cells inside the wall. The velocity components on these cells are linearly extrapolated from the computational cells inside the domain. The temperature is also linearly extrapolated using the specified wall temperature. The density is calculated from the equation of state considering zero pressure gradient normal to the wall. We apply supersonic outflow conditions at the outlet and far-field boundaries. Furthermore, we use a synthetic turbulent inflow boundary condition to produce a freestream flow with a superimposed random turbulence.

Table 1

Simulation parameters

\begin{tabular}{llllllllll}
\hline$\delta(\mathrm{mm})$ & $U_{\infty}(\mathrm{m} / \mathrm{s})$ & $P_{\infty}(\mathrm{Pa})$ & $T_{\infty}(\mathrm{K})$ & $M_{\infty}$ & $\rho_{\infty}\left(\mathrm{kg} / \mathrm{m}^{3}\right)$ & $T_{w}(\mathrm{~K})$ & $R e_{\delta}$ & $R e_{\theta}$ & $R e_{\tau}$ \\
\hline 5.0 & 1,146 & $1,365.6$ & 63.06 & 7.2 & 0.0755 & 340 & 102,731 & 3,500 & 198 \\
\hline
\end{tabular}

The synthetic turbulent inflow boundary condition is based on the digital filter (DF) method documented in [39, $40,41]$ and, specifically validated in the framework of the code CNS3D in [42, 43, 44, 27]. According to DF, instead of using a white-noise random perturbation at the inlet, energy modes within the Kolmogorov inertial range scaling with $k^{-5 / 3}$, where $\mathrm{k}$ is the wavenumber, are introduced into the turbulent boundary layer. Opposite to the von Kármán atmospheric spectrum introduced at the inlet of the first case, the DF does not introduce any large-scale energy modes scaling with $k^{4}$. A cutoff at the maximum frequency of $50 \mathrm{MHz}$ is applied, since the finest mesh used in this study would under-resolve higher values. The turbulence intensity at the inlet $(T u)$ is set as $3 \%$ of the intensity of the freestream velocity.

Following typical resolution recommendations for LES and DNS simulations $[45,46,10]$ three relative fine meshes were used in this study ranging from fine wall-resolved LES (G1) to under-resolved DNS (G3). We cluster the mesh 


\section{Table 2}

Mesh parameters

\begin{tabular}{lllllllll}
\hline & $N_{x}$ & $N_{y}$ & $N_{z}$ & $\Delta x_{\min }^{+}$ & $\Delta x_{\max }^{+}$ & $\Delta y_{w}^{+}$ & $\Delta y_{e}^{+}$ & $\Delta z^{+}$ \\
\hline G1 & 401 & 167 & 107 & 11.48 & 41.14 & 1.0 & 7.37 & 13.48 \\
G2 & 601 & 249 & 161 & 7.7 & 27.35 & 1.0 & 4.26 & 8.96 \\
G3 & 801 & 333 & 213 & 5.77 & 20.4 & 0.5 & 3.65 & 6.77 \\
LES[45, 46, 10] & - & - & - & 50 & 150 & $\leq 1.0$ & - & $15-40$ \\
DNS[45, 46, 10] & - & - & - & 10 & 20 & $<1.0$ & - & $5-10$ \\
\hline
\end{tabular}

near the corner in the stream-wise direction and near the wall in the wall-normal direction. We summarize the number of mesh points and the mesh spacing in Table 2 along with the LES and DNS recommendations from the literature. The present mesh spacing $(\Delta y)$ is scaled using the conventional inner variable method $\Delta y^{+}=u_{\tau} \Delta y / v_{w}$, where $u_{\tau}=$ $\sqrt{\tau_{w} / \rho_{w}}$ is the friction velocity, $v_{w}$ is the near wall kinematic viscosity, $\tau_{w}$ is the near wall shear stress, and $\rho_{w}$ is the near wall density. We performed a mesh convergence study in [23] with the two finer meshes, G2 and G3, showing close agreement in all calculated quantities and justifying the use of G3 in the analysis below.

We visualise the structure of turbulence at the compression/expansion ramp by iso-surfaces based on the compressible Q-criterion [47, 48] Figure 4. The iso-surfaces are coloured with the stream-wise velocity showing the flow separation at the corner. The density gradient is also plotted in grey-scale indicating the position and the thickness of the shock. The flow statistics are computed by averaging in time over seven flow-cycles and spatially in the span-wise direction. The total simulation time for each case is equal to at least twelve flow-cycles with the first five omitted from the calculations for statistical purposes. The statistical convergence of the simulations is assessed by comparing the results between fifteen and seven flow-cylces with mesh G1 resulting in less than $2 \%$ difference.

For a better visualisation of the flow separation we show the flowfield streamlines over a temperature contour plot in Figure 5. In the re-circulation region the temperature is significantly higher compared to the free-stream conditions reaching a maximum of $570 \mathrm{~K}$ at the re-attachment point. In the following section we will show how the various changes in the flow field (separation, re-circulation, re-attachment, expansion, etc.) are affecting not only the magnitude of the acoustic loading but also its spectral characteristics.

The density gradients in Figure 4 indicated the position and thickness of the lambda shock. In Figure 6 we present the magnitude of the pressure gradient $(|\nabla P|)$ averaged in the spanwise direction and in time. The lambda shock and two high pressure gradients regions, are shown. The first region is the expansion-fan created at the expansion corner of the ramp. The second region is above the re-attachment of the flow on the ramp, very close to the wall. This area is highlighted in inset (b) of Figure 6 where the red colour indicates the maximum values of pressure gradient calculated near the wall.

Laganelli et al. [1] proposed a theoretical model for $P_{r m s}^{\prime} / q_{\infty}$ beneath compressible fully turbulent attached bound- 


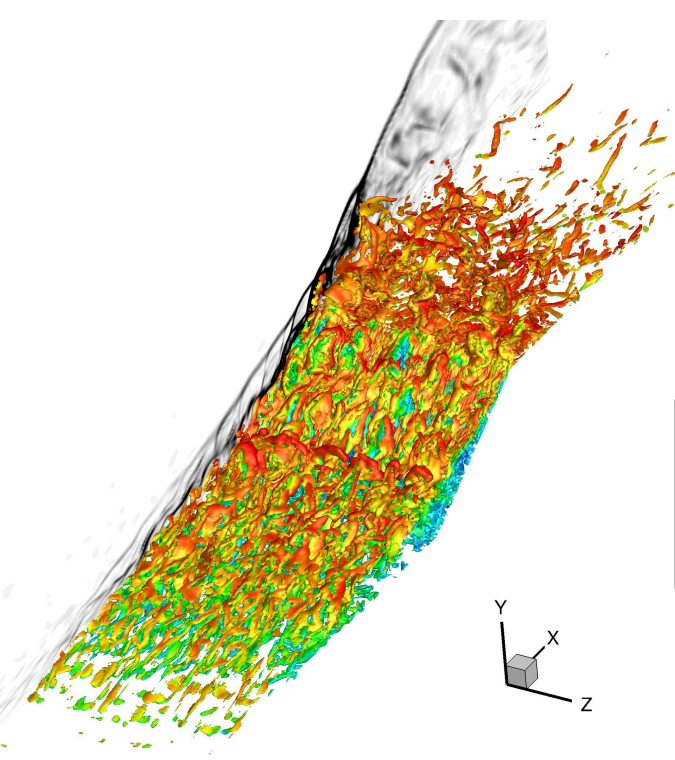

(a)

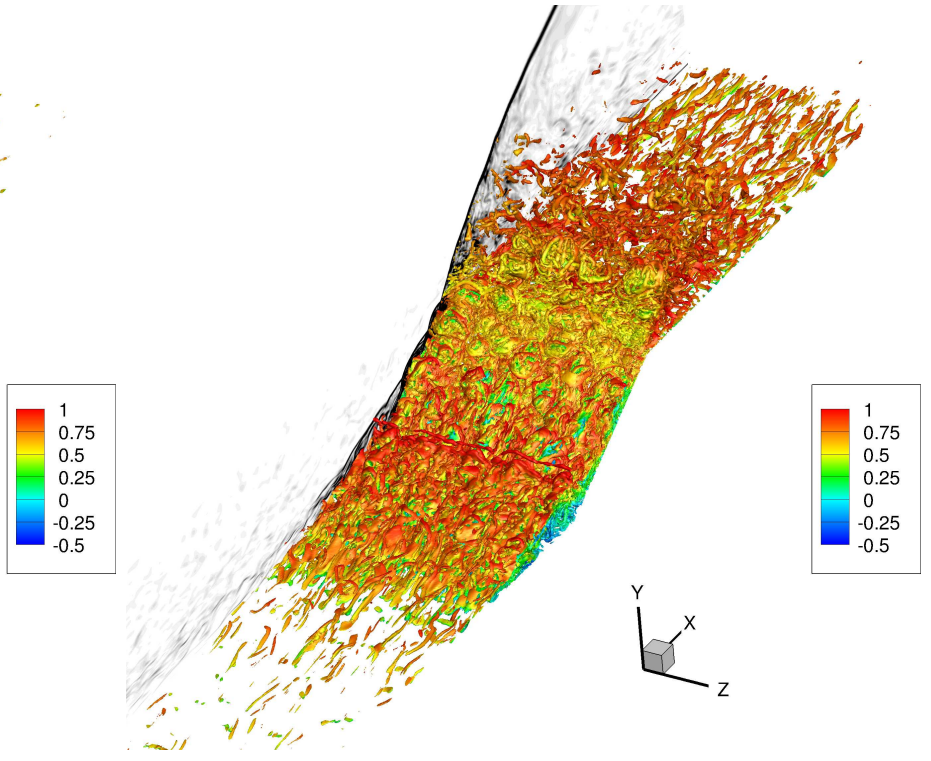

(b)

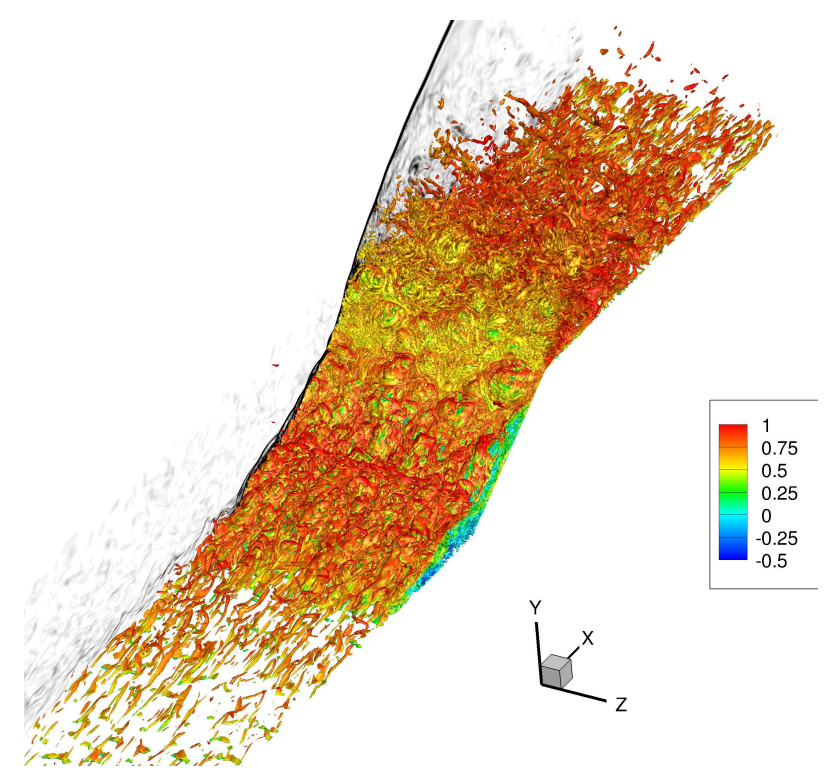

(c)

Figure 4: Isosurfaces of compressible Q-criterion coloured by streamwise velocity indicating the flow separation at the corner. The density gradient is also plotted in grayscale at the side of the plot highlighting the position and the thickness of the shock.

ary layers, where $q_{\infty}=\left(\rho_{\infty} u_{\infty}^{2}\right) / 2$ is the dynamic pressure and $u_{\infty}$ is the free-stream velocity. The model is based on fitting incompressible measurements $[49,50,51,52]$ to compressible flows by taking into account the wall temperature and free-stream Mach number:

$$
\frac{P_{r m s}^{\prime}}{q_{\infty}}=\frac{0.006}{\left[0.5+\left(T_{w} / T_{a w}\right)\left(0.5+0.09 M_{\infty}^{2}\right)+0.04 M_{\infty}^{2}\right]^{0.64}},
$$




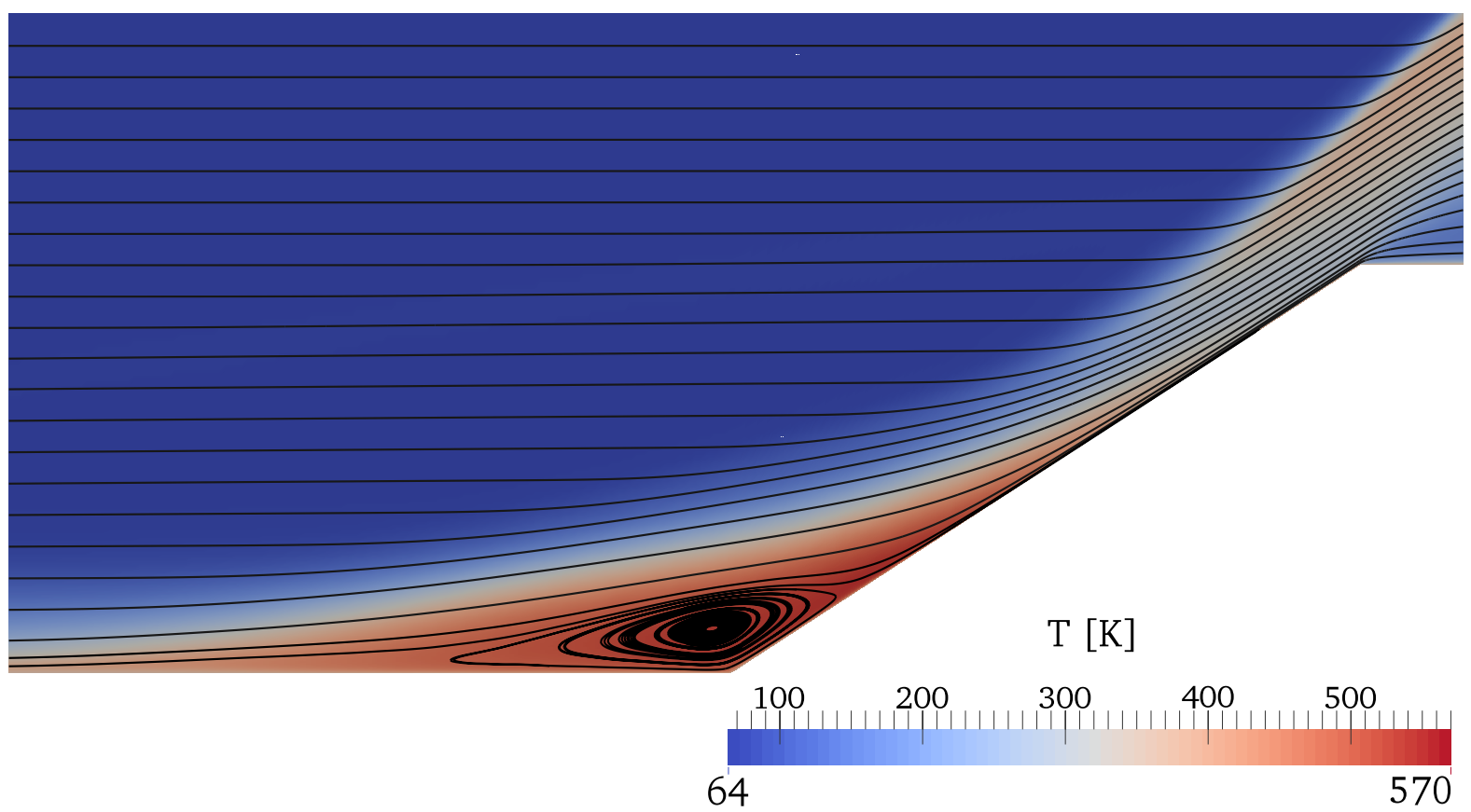

Figure 5: Flowfield streamlines and temperature contour plot in the region of the compression/expansion ramp. All variables have been averaged over time and in the spanwise direction.

where $T_{a w}$ is the adiabatic wall temperature calculated from the recovery temperature. Previous studies $[8,53,54,55]$ have suggested that the value in the numerator of Eq. 3 should be between 0.008 and 0.010, with [6] suggesting an $R e_{\theta}$ dependence. Beresh et al. [8] has proposed a value of 0.009 for the incompressible limit based on an estimated extension of the measured pressure spectra. According to the simulations in [25], a value of 0.008 is suggested for flows up to Mach 8. The theoretical model with the suggested value (dashed black line in inset (a) of Figure 6) shows good agreement with the calculated values in the areas of the domain where the flow is attached and the pressure gradients are low. In the recirculation region the (normalised) pressure fluctuations increase and extremely high values are recorded after the re-attachment of the flow where the maximum pressure gradients are also observed (Insets (a) and (b) of figure 6). This is a strong indication that acoustic loading is correlated with areas of high pressure gradients.

\section{Acoustic loads}

We performed acoustic loading calculations at various locations of a compression/expansion corner under hypersonic conditions. Furthermore, we compared these results with calculations beneath hypersonic transitional and turbulent boundary layers over sa flat panel. From the analysis in the previous section, acoustic loads are expected to peak at the re-attachment point, obtaining significantly higher values than those sustained in the rest of the domain. A representative quantity of acoustic loading is the overall Sound Pressure Level (SPL). This quantity can be obtained through the transformation of pressure fluctuations, SPL $=20 \log _{10}\left(P^{\prime} / P_{0}\right) \mathrm{dB}$, where $P_{0}=20 \mu \mathrm{Pa}$. 


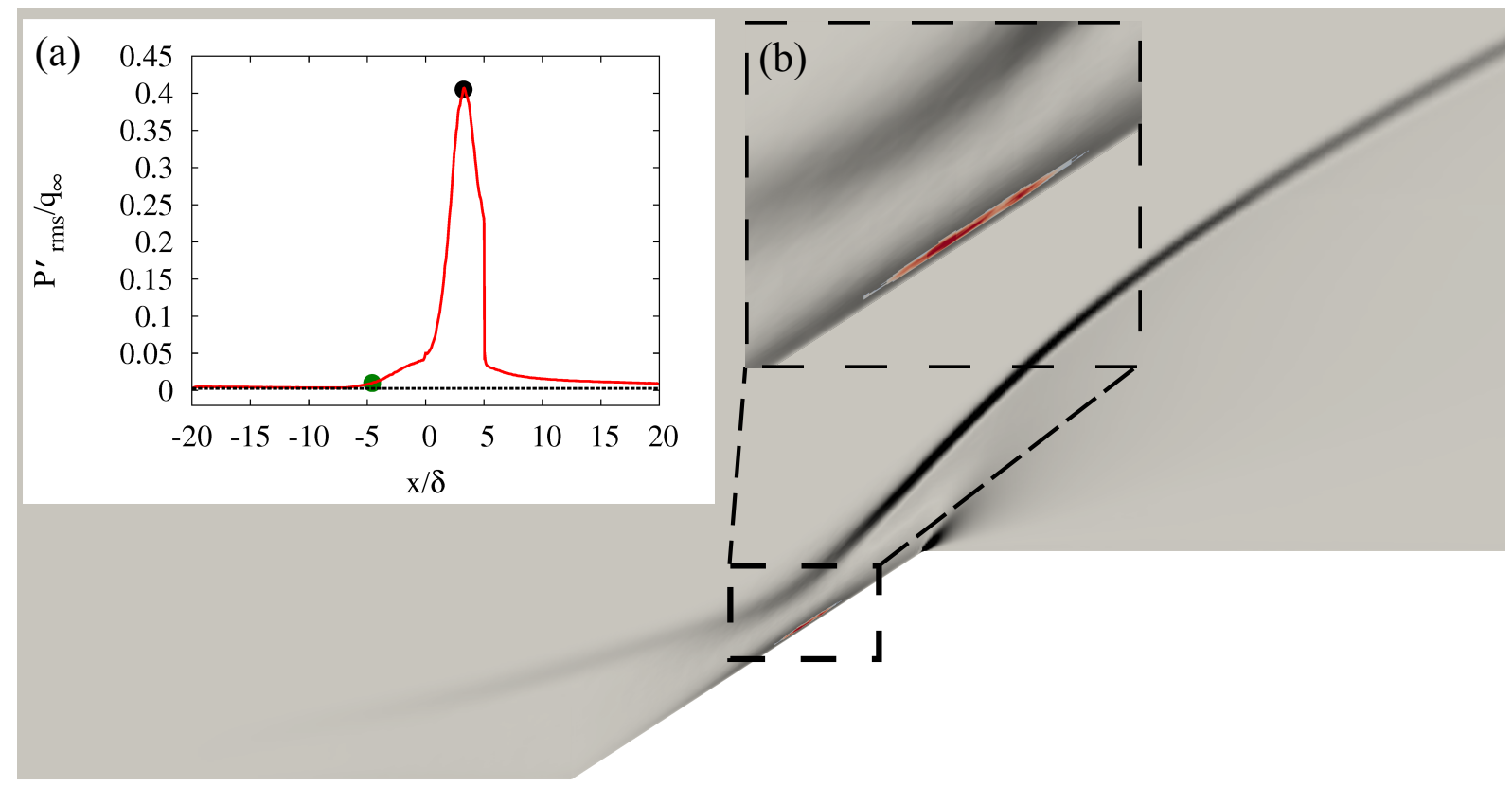

Figure 6: Magnitude of pressure gradient $(|\nabla P|)$ over the simulation domain. Darker (black) areas indicate higher $|\nabla P|$. Inset $(a)$ is the root mean square of wall pressure fluctuations $\left(P_{r m s}^{\prime}\right)$ along the wall normalised by the freestream dynamic pressure, $q_{\infty}$. The black dashed line represent theoretical predictions based on Eq. 3 with $T_{w} / T_{a w}=0.524$; see discussion in the text. The green filled circle is the foot of the recirculation bubble. The black filled circle is the point of maximum $|\nabla P|$. Inset (b) magnifies the area of the highest $|\nabla P|$ on the wall. Red colour in that case indicates high $|\nabla P|$.

The re-attachment point, as well as the exact magnitude of the acoustic load, are important for the structural design of hypersonic vehicles. In Figure 7 we show the SPL over the whole simulation domain, highlighting the re-attachment region where values above $170 \mathrm{~dB}$ are observed, significantly higher than the $140 \mathrm{~dB}$ observed in the TBL region before the separation bubble and the $145 \mathrm{~dB}$ after the expansion corner. In the same figure we also show five positions that we selected for further analysis. These points are representative of the different flowfield conditions encountered in this simulation. More specifically the first position is away from the compression ramp and before the recirculation bubble where the flow can be considered attached and fully turbulent. The second location is close to the foot of the lambda shock. The pressure history calculations also show the shock oscilaltions. The third position is in the recirculation bubble where the flow is detached, while the fourth position is close to the re-attachment point where maximum SPL values where calculated. Finally, the last position is just after the expansion fan where the flow is attached and fully turbulent but under different conditions compared to the first position.

In Figures 8(a) to 8(e) we show the normalised pressure histories at the selected locations, while in Figures 8(f) and $8(\mathrm{~g})$ we compare the pressure histories from the first three and the last three positions, respectively. The pressure histories are recorded from the $5^{\text {th }}$ until the $12^{\text {th }}$ flow cycle $\left(t_{c}\right)$. The lowest pressures are recorded at the first position (Figure 8(a)), while the highest at the fourth position (Figure $8(\mathrm{~d})$ ) where the flow is re-attached. The calculated pressures close to the re-attachment point are at least an order of magnitude higher than those calculated at other 


\section{$\mathrm{SPL}(\mathrm{dB})$}

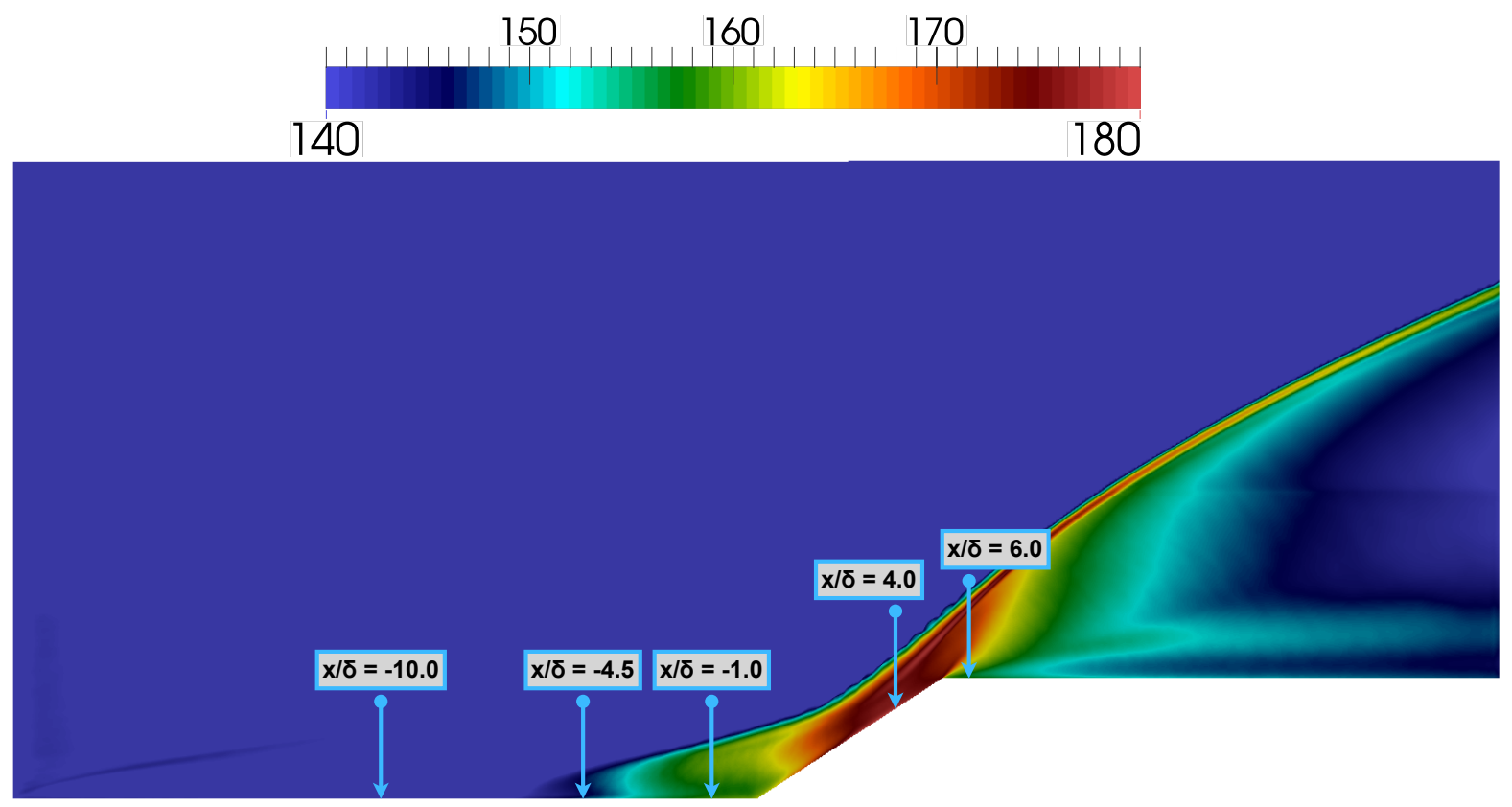

Figure 7: Sound pressure levels contour plot and locations of spectral analysis.

locations (for comparison see Figures 8(f) and 8(g)). The pressure history of the second position (Figure 8(b)) exhibits values much higher than the average ones in periodic intervals. We attribute the high pressure values to the oscillation of the lambda shock around this location.

While the calculation of pressure and pressure fluctuations are indicative of the acoustic loading imposed on the wall, it is equally important to study the frequency content of these fluctuations. We use the Welch method [56] to calculate the power spectral density (PSD) of the pressure fluctuations in specific locations. For the compression/expansion corner flow the sampling frequency is approximately $16 \mathrm{MHz}$, and the overall pressure record is subdivided into 8 segments, each including $2 \times 1,200$ samples; note that we observe a negligible difference between the PSD produced using 8 and 12 segments. Details regarding the PSD calculation approach on the flat plate can be found in [25]. The present spectral analysis of the pressure fluctuations on the wall is based on the single-point spectrum in the frequency domain which is defined as

$$
\Phi(\omega)=\frac{1}{2 \pi} \int_{-\infty}^{\infty} \overline{P^{\prime}(x, y, z, t) P^{\prime}(x, y, z, t+\tau)} e^{-i \omega \tau} d \tau,
$$

where $\tau$ is a time delay and $\omega$ is the radial frequency.

The spectral behaviour of attached fully turbulent boundary layers in both subsonic and supersonic flows has been studied extensively. Bull [53] isolated four different regions of low, mid (which includes the spectral peak), mid- 

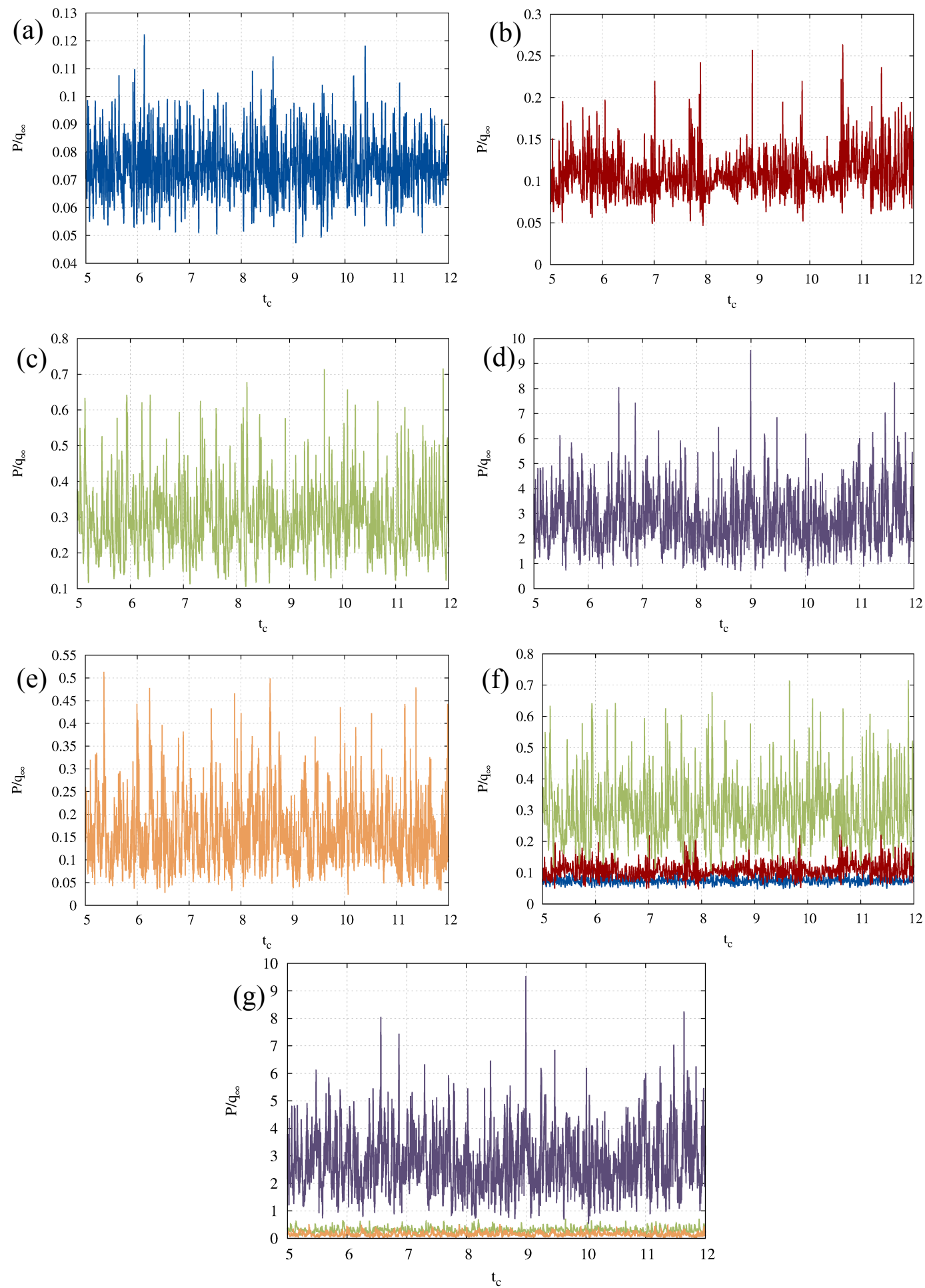

Figure 8: Pressure history at various locations on the ramp wall. Pressure is normalised by the dynamic freestream pressure $\left(q_{\infty}\right)$ and $t_{c}$ is the time normalised by the time needed for one flow cycle. The pressure histories correspond to the following locations: (a) $x / \delta=-10$, (b) $x / \delta=-4.5$, (c) $x / \delta=-1$, (d) $x / \delta=4$, and (e) $x / \delta=6$. (f) shows a comparison of the pressure histories in the first three locations, while $(\mathrm{g})$ for the last three locations. 
to-high overlap, and high frequencies, with corresponding spectrum slopes of $\omega^{2}, \omega^{0}, \omega^{-r}(r=[0.7,1.1])$, and $\omega^{-t}$ $(t=[7 / 3,5])$, respectively. The turbulent motion influences the low frequency region in the outer part of the boundary layer. In contrast, high frequencies are influenced by the viscosity and turbulent motion in the inner part of the boundary layer.

The spectrum roll-off at the same locations of the compression/expansion ramp where the pressure histories are shown in Figure 9. Also, we show the spectrum roll-off at two locations of the boundary layer over the flat plate, one at the end of the transition region and one in the fully turbulent region (Figure 10).

According to the theoretical arguments made by Ffowcs-Williams [2] for compressible flows, in the low frequency region the scaling of $\omega$ should approach 0 . Experimental and numerical studies of supersonic and hypersonic turbulent boundary layers have confirmed the above observation $[22,57,8,9]$. The above scaling is in contrast to the KraichnanPhillips theorem for incompressible flows [53, 58, 59], which suggests $\omega^{2}$. In our calculations the low frequency region yields $\omega^{f \rightarrow 0}$ which agrees with the analysis above. The only exception to this scaling is shown in the spectrum roll-off at the end of the transition region on the flat plate (Figure 10(a)) where the simulations yield $\omega^{0.3}$. The comparison between the simulation results of the flat plate and upstream region of the ramp (before the occurrence of separation) shows similarities at higher frequencies, e.g., regions of $\omega^{-5}$ and $\omega^{-9}$ in Figures 9(c))a and (Figure 10b. However, there are also differences in how the spectrum develops in the $\omega^{-1.3}$ and $\omega^{-7.3}$ regions of the spectrum. We believe that the separation around the corner of the ramp influences the spectrum results of the position $x / \delta=-10$, which is in the proximity.

The mid-to-high overlap frequency region appears at sufficiently high $R e_{\theta}$ values and the spectrum varies as $\omega^{-r}$ with $r=0.7$ to 1.1 , influenced by the local Reynolds number. This region is associated with pressure-induced eddies in the logarithmic region of the boundary layer. Bradshaw [3] predicted its scaling behaviour, and was verified theoretically [60] and experimentally $[8,6]$. This frequency region can be identified in all but one calculation point on the ramp, as well as in the fully turbulent region of the flat plate. The value of $r$ lies between 1.0 to 1.3 depending on the location. The calculations at the foot of the lambda shock (Figure 9(c)) did not produce this scaling region, while the scaling at the end of the transition region is $\omega^{-0.5}$. In the present calculations the first two positions produced a sudden spectrum rise with a scaling up to $\omega^{2}$ in a short frequency range. We believe that this frequency increase is related to the incoming turbulence produced by the lambda shock.

Following the mid-to-high overlap frequency region, the spectrum becomes $\omega^{-7 / 3}$, henceforth called "acoustictransition", which is in accordance to the isotropic turbulence theory of Batchelor [61]. Various experiments [62, $63,64]$ have shown the above spectrum and there is further verification through numerical calculations of supersonic turbulent boundary layers [22,9]. This "acoustic-transition" region is present in all the calculated spectra. It is also identified in the spectra inside the re-circulation bubble (Figure 9(c)) and close to the re-attachment point (Figure 9(d)). 


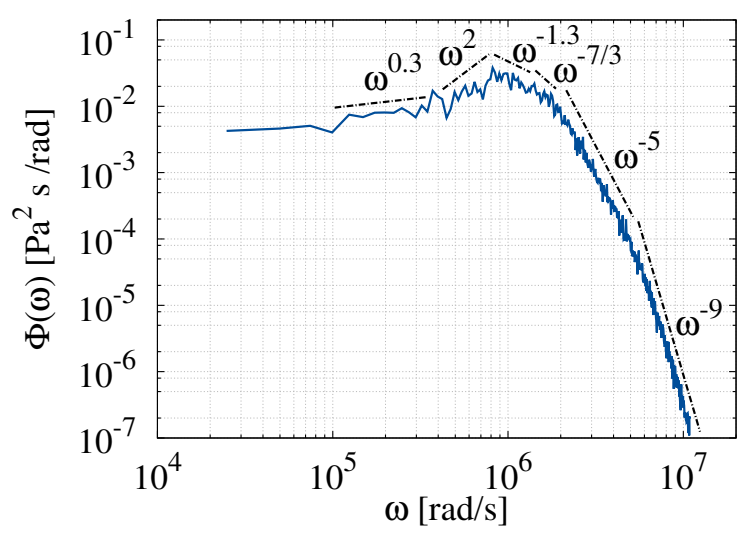

(a) $x / \delta=-10$

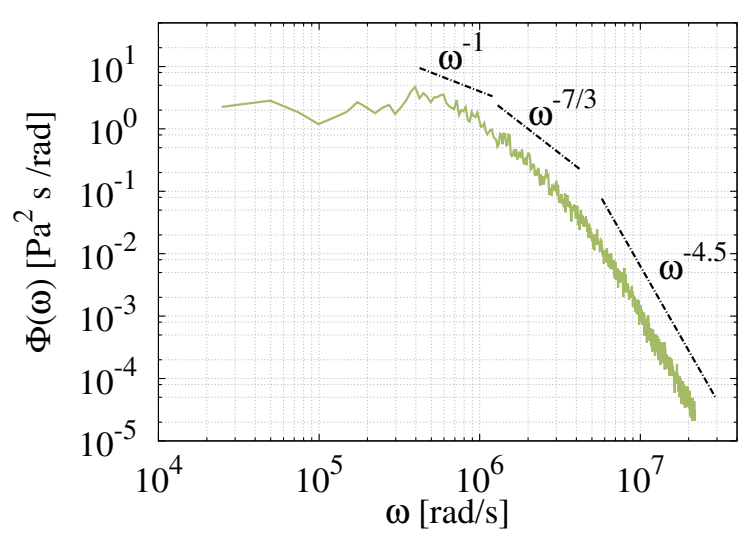

(c) $x / \delta=-1.0$

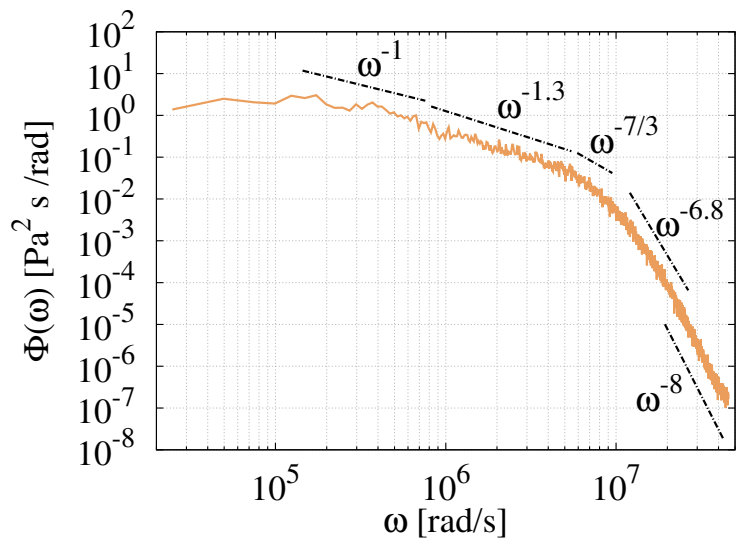

(e) $x / \delta=6.0$

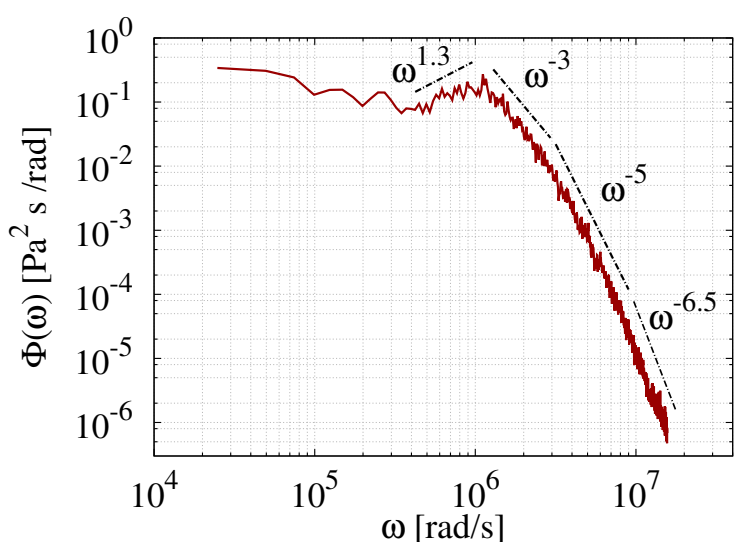

(b) $x / \delta=-4.5$

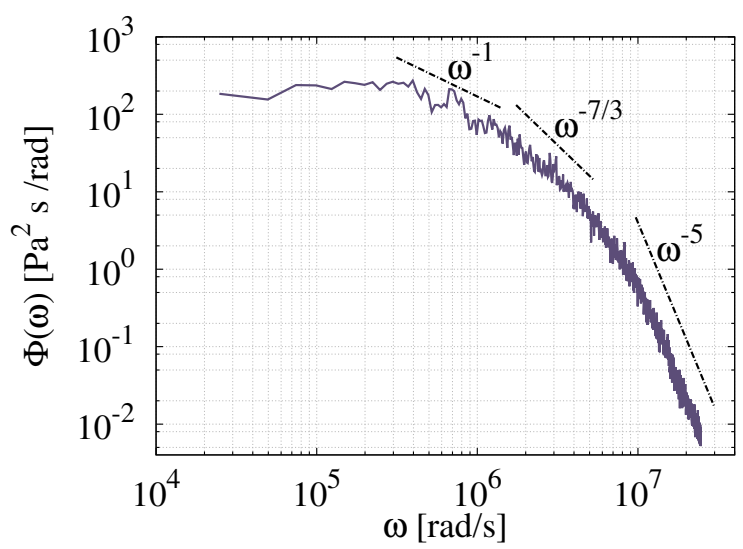

(d) $x / \delta=4.0$

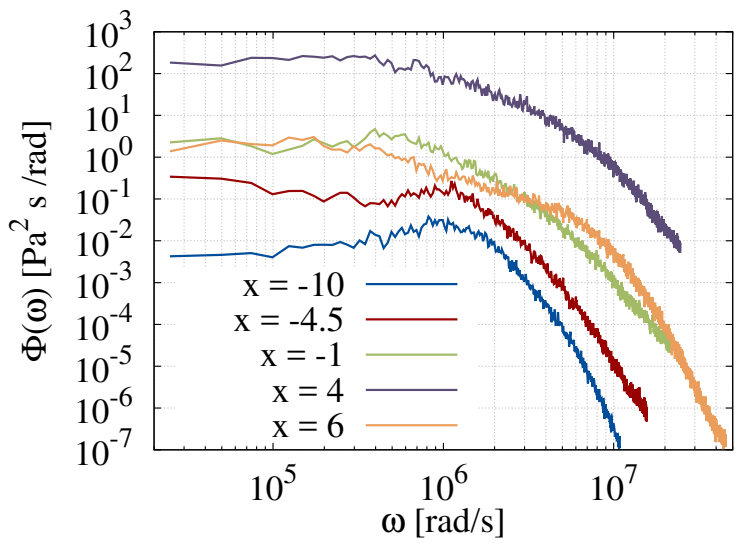

(f) All positions

Figure 9: PSD at various locations on the compression/expansion corner. Figure $(f)$ is comparison of the spectra among all the locations under consideration. 


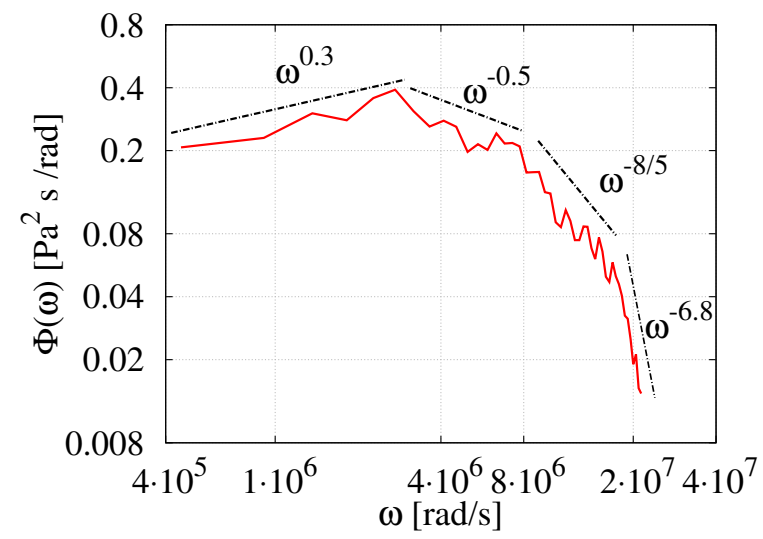

(a) End of the transition region

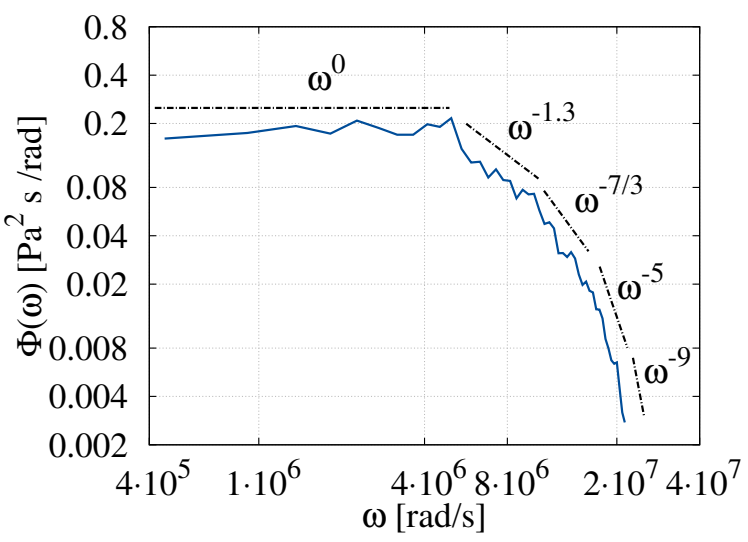

(b) Fully turbulent region

Figure 10: PSD at two locations beneath the hypersonic boundary layer over a flat plate.

The scaling of this frequency region is slightly steeper $\left(\omega^{-3}\right)$ in the spectrum calculated beneath the foot of the lambda shock. At the same time, it is shallower $\left(\omega^{-8 / 5}\right)$ in the spectrum calculated at the end of the transition region. We believe that the oscillating shock and turbulence bursts, for the compression/expansion ramp and the flat plate, respectively, are responsible for the slope change in this frequency region. The theoretical prediction for this frequency region by Batchcelor is based on the assumption of isotropic turbulence. The deviation of the scaling behaviour from $\omega^{-7 / 3}$ may be due to the onset of flow anisotropy and localised coherent structures; however, this point requires further investigation.

At high frequencies the spectrum decays more rapidly reaching a slope proportional to $\omega^{-5}$. Sources in the sublayer $\left(y^{+}<20\right)$ contribute to this frequency region according to the theoretical prediction of Blake [4], with the scaling validated experimentally $1[6,5]$. This frequency region appears in all calculations. In two cases the slope is steeper at $\omega^{-6.8}$; These are the positions close to the expansion fan in the case of the ramp and the transition region in the case of the flat plate, respectively.

Additionally, in some locations on the ramp (see Figures 9(a), 9(b) and 9(e)) and also in the fully turbulent region of the flat plate the last leg of the high frequency encompasses a region of (approximately) $\omega^{-8}$. We attribute this region to high-speed, compressibility effects closer to the wall $\left(y^{+}<20\right)$. Experiments [65] and simulations [22] also observed a Mach number dependence of the spectrum in fully turbulent boundary layers that lead to steeper gradients.

\section{Conclusions}

We have investigated acoustic effects beneath hypersonic transitional and turbulent boundary layers by performing spatial and spectral analysis of near-wall pressure fluctuations. Simulations of two different flows, one over a flat 
plate at Mach 6 and one over a compression/expansion corner at Mach 7.2, have been carried out. We have validated simulations against theoretical, DNS and experimental data, where available. The most important conclusions drawn from the present study are summarised below:

- The modified Laganelli's theoretical model is valid for fully turbulent attached boundary layers but underpredicts the pressure fluctuations beneath re-circulations or areas of high pressure gradients.

- High SPL values are associated with shock re-attachment and structural panels will be subjected to the strongest acoustic fatigue in this region.

- Spectral analysis was performed at various locations of the compression/expansion ramp and compared with calculations at points of the flow over a flat plate. This analysis highlighted similarities between the transition region on the flat plate and the point beneath the lambda shock on the expansion/compression ramp, where turbulence bursts and shock motion, respectively, alter the spectrum shape from the one expected beneath turbulent boundary layers.

- The pressure fluctuations calculated on the compression/expansion ramp are governed by scaling laws that are generally in agreement with the ones in fully turbulent flows.

- In areas where the boundary layer is attached, a region of very high frequencies with a slope proportional to $\omega^{-8}$ was observed. This region is related to high-speed, compressibility effects near the wall.

\section{Acknowledgments}

This work was sponsored by the Air Force Office of Scientific Research, Air Force Material Command, USAF, under grants number FA9550-14-1-0224 and FA9550-19-1-7018. The U.S. Government is authorised to reproduce and distribute reprints for Governmental purpose notwithstanding any copyright notation thereon. The authors would like to thank Z. Riley and D. Garner for their support. The authors would also like to thank EPSRC for providing access to computational resources on the National HPC facility ARCHER (http://www.archer.ac.uk) through the UK Applied Aerodynamics Consortium Leadership Project “e529”.

\section{References}

[1] A. L. Laganelli, A. Martellucci, L. L. Shaw, Wall pressure fluctuations in attached boundary-layer flow, AIAA Journal 21 (1983) $495-502$.

[2] J. E. Ffowcs-Williams, Surface pressure fluctuations induced by boundary layer flow at finite Mach number, J. Fluid Mech. 22 (1965) $507-519$.

[3] P. Bradshaw, Inactive motion and pressure fluctuations in turbulent boundary layers, J. Fluid Mech. 30 (1967) $241-258$.

[4] W. K. Blake, Mechanics of Flow-Induced Sound and Vibration, New York: Academic Press, 1986. 
[5] T. M. Farabee, M. J. Casarella, Spectral features of wall pressure fluctuations beneath turbulent boundary layers, Phys. Fluids A 3 (1991) $2410-2420$

[6] S. P. Gravante, A. M. Naguip, C. E. Wark, H. M. Nagib, Characterization of the pressure fluctuations under a fully developed turbulent boundary layer, AIAA Journal 36 (1998) 1808-1816.

[7] Y. Tsuji, J. H. N. Fransson, P. H. Alfredsson, A. V. Johansson, Pressure statistics and their scaling in high-reynolds-number turbulent boundary layers, J. Fluid Mech. 585 (2007) 1-40.

[8] S. J. Beresh, J. F. Henfling, R. W. Spillers, B. O. M. Pruett, Fluctuating wall pressures measured beneath a supersonic turbulent boundary layer, Phys. Fluids 23 (2011) 1-16.

[9] M. Bernardini, S. Pirozzoli, Wall pressure fluctuations beneath supersonic turbulent boundary layers, Phys. Fluids 23 (2011) 1-11.

[10] J. Poggie, N. J. Bisek, R. Gosse, Resolution effects in compressible, turbulent boundary layer simulations, Comput. Fluids 120 (2015) 57-69.

[11] S. M. Spottswood, T. J. Beberniss, T. G. Easona, R. A. Perez, J. M. Donbar, D. A. Ehrhardt, Z. B. Riley, Exploring the response of a thin, flexible panel to shock-turbulent boundary-layer interactions, Journal of Sound and Vibration 443 (2019) 74-89.

[12] Z. B. Riley, R. A. Pereza, G. W. Bartrama, S. M. Spottswood, B. P. Smarslok, T. J. Beberniss, Aerothermoelastic experimental design for the aedc/vkf tunnel c: Challenges associated with measuring the response of flexible panels in high-temperature, high-speed wind tunnels, Journal of Sound and Vibration 441 (2019) 96-105.

[13] R. Wiebe, S.M.Spottswood, On the dimension of complex responses in nonlinear structural vibrations, Journal of Sound and Vibration 373 (2016) 192-204.

[14] C. M. Helm, M. P. Martín, New LES of a Hypersonic Shock/Turbulent Boundary Layer Interaction, in: 54th AIAA Aerospace Sciences Meeting, 2016, pp. 1-15.

[15] C. M. Helm, P. M. Martin, Görtler-like vortices in the LES Data of a mach 7 STBLI, in: 55th AIAA Aerospace Sciences Meeting, 2017, pp. $1-13$.

[16] V. Mikulla, C. C. Horstmann, Turbulence measurements in hypersonic shock-wave boundary layer interaction flows, AIAA Journal 14 (1976) $568-575$.

[17] P. Bookey, C. Wyckham, A. J. Smits, M. P. Martín, New experimental data of stbli at dns/les accesible Reynolds numbers, in: 43rd AIAA Aerospace Sciences Meeting and Exhibit, AIAA 2005-309, 2005, pp. 1-18.

[18] A. M. Schreyer, D. Sahoo, A. J. Smits, Experimental investigations of a hypersonic shock turbulent boundary layer interaction, in: 49th AIAA Aerospace Sciences Meeting, AIAA 2011-481, 2011, pp. 1-12.

[19] A. M. Schreyer, D. Sahoo, A. J. Smits, Turbulence measurements with PIV in a hypersonic shock boundary layer interaction, in: 41st Fluid Dynamics Conference and Exhibit, AIAA 2011-3429, 2011, pp. 1-14.

[20] L. Duan, I. Beekman, M. P. Martín, Direct numerical simulation of hypersonic turbulent boundary layers. part 3. effect of mach number, J. Fluid Mech. 672 (2011) 245-267.

[21] K. J. Franko, S. K. Lele, Breakdown mechanisms and heat transfer overshoot in hypersonic zero pressure gradient boundary layers, J. Fluid Mech. 730 (2013) 491-532.

[22] L. Duan, M. M. Choudhari, C. Zhang, Pressure fluctuations induced by a hypersonic turbulent boundary layer, J. Fluid Mech. 804 (2016) $578-607$.

[23] K. Ritos, I. W. Kokkinakis, D. Drikakis, Physical insight into a mach 7.2 compression corner flow, in: 2018 AIAA Aerospace Sciences Meeting, 2018. doi:10.2514/6.2018-1810.

[24] K. Ritos, I. W. Kokkinakis, D. Drikakis, Performance of high-order implicit large eddy simulations, Comput. Fluids 173 (2018) 307 - 312. 


\section{Aeroacoustic Loading in high-speed TBL}

[25] K. Ritos, D. Drikakis, I. W. Kokkinakis, Acoustic loading beneath hypersonic transitional and turbulent boundary layers, J. Sound Vib. 441 (2019) 50-62.

[26] D. Drikakis, M. Hahn, A. Mosedale, B. Thornber, Large eddy simulation using high resolution and high order methods, Proc. R. Soc. A 367 (2009) 2985-2997.

[27] K. Ritos, I. W. Kokkinakis, D. Drikakis, S. M. Spottswood, Implicit large eddy simulation of acoustic loading in supersonic turbulent boundary layers, Phys. Fluids 29 (2017) 1-11.

[28] K. Ritos, I. W. Kokkinakis, D. Drikakis, Physical insight into the accuracy of finely-resolved iLES in turbulent boundary layers, Comput. Fluids 169 (2018) 309-316.

[29] E. F. Toro, M. Spruce, W. Speares, Restoration of the contact surface in the hll-riemann solver, Shock Waves 4 (1994) 25-34.

[30] E. F. Toro, Riemann Solvers and Numerical Methods for Fluid Dynamics, 3rd ed., Springer, 2009.

[31] D. S. Balsara, C. W. Shu, Monotonicity preserving weighted essentially non-oscillatory schemes with increasingly high order of accuracy, J. Comput. Phys. 160 (2000) 405-452.

[32] R. Spiteri, S. J. Ruuth, New class of optimal high-order strong-stability-preserving time discretization methods, SIAM J. Numer. Anal. 40 (2002) 469-491.

[33] L. Duan, M. M. Choudhari, Numerical study of pressure fluctuations due to a mach 6 turbulent boundary layer, in: 51st AIAA Aerospace Sciences Meeting, 2013, pp. 1-16.

[34] S. Stolz, N. A. Adams, Large-eddy simulation of high-reynolds-number supersonic boundary layers using the approximate deconvolution model and a rescaling and recycling technique, Phys. Fluids 15 (2003).

[35] S. Stolz, High-pass filtered eddy-viscosity models for large-eddy simulations of compressible wall-bounded flows, J. Fluids Eng 127 (2005) 666-673.

[36] I. Marusic, B. J. McKeon, P. A. Monkewitz, H. M. Nagib, A. J. Smits, K. R. Sreenivasan, Wall-bounded turbulent flows at high reynolds numbers: Recent advances and key issue, Phys. Fluids 22 (2010).

[37] T. Gatski, J.-P. Bonnet, Compressibility, Turbulence and High Speed Flow, Academic Press, 2013.

[38] J. Blazek, Fluid Dynamics, Principles and Applications, Elsevier, 2015.

[39] T. S. Lund, X. Wu, K. D. Squires, Generation of turbulent inflow data for spatially-developing boundary layer simulations, J. Comput. Phys. 140 (1998) 233-258.

[40] M. Klein, A. Sadiki, J. Janicka, A digital filter based generation of inflow data for spatially developing direct numerical simulation or large eddy simulations, J. Comput. Phys. 186 (2003) 652-665.

[41] E. Touber, N. D. Sandham, Large-eddy simulation of low-frequency unsteadiness in a turbulent shock-induced separation bubble, Theor. Comp. Fluid Dyn. 23 (2009) 79-107.

[42] D. Drikakis, Advances in turbulent flow computations using high-resolution methods, Prog. Aerosp. Sci. 39 (2003) $405-424$.

[43] Z. A. Rana, B. Thornber, D. Drikakis, Transverse jet injection into a supersonic turbulent cross-flow, Phys. Fluids 23 (2011) 1-21.

[44] Z. A. Rana, B. Thornber, D. Drikakis, On the importance of generating accurate turbulent boundary condition for unsteady simulations, J. Turbul. 12 (2011) 1-39.

[45] N. J. Georgiadis, D. P. Rizzetta, C. Fureby, Large-eddy simulation: current capabilities, recommended practices, and future research., AIAA Journal 48 (2010) 1172-1784.

[46] H. Choi, P. Moin, Grid-point requirements for large eddy simulation: Chapman's estimates revisited, Phys. Fluids 24 (2012) 011702.

[47] V. Kolář, Compressibility effect in vortex identification, AIAA Journal 47 (2009) 473-475. 
[48] V. Kolář, J. Šístek, Corotational and compressibility aspects leading to a modification of the vortex-identification q-criterion, AIAA Journal 53 (2015) 2406-2410.

[49] R. D. Shattuck, Sound pressures and correlations of noise on the fuselage of a jet aircraft in flight, Technical Report TN-D-1086, NASA, 1961.

[50] W. W. Willmarth, C. E. Wooldridge, Measurements of the fluctuating pressure at the wall beneath a thick turbulent boundary layer, J. Fluid Mech. 14 (1962) 187-210.

[51] W. W. Willmarth, F. W. Roos, Resolution and structure of the wall pressure field beneath a turbulent boundary layer, J. Fluid Mech. 22 (1965) 81-94.

[52] M. K. Bull, Wall-pressure fluctuations associated with subsonic turbulent boundary layer flow, J. Fluid Mech. 28 (1967) $719-754$.

[53] M. K. Bull, Wall-pressure fluctuations beneath turbulent boundary layers: Some reflections on forty years of research, J. Sound Vib. 190 (1996) 299-315.

[54] R. M. Lueptow, Transducer resolution and the turbulent wall pressure spectrum, J. Acoust. Soc. Am. 97 (1995) $370-378$.

[55] M. C. Goody, R. L. Simpson, Surface pressure fluctuations beneath two= and three-dimensional turbulent boundary layers, AIAA Journal 38 (2000) 1822-1831.

[56] P. D. Welch, The use of Fast Fourier Transform for the estimation of power spectra: A method based on time averaging over short, modified periodograms, IEEE Trans. Audio Electroacoust. AU-15 (1967) 70-73.

[57] C. Zhang, L. Duan, M. M. Choudhari, Effect of wall cooling on boundary-layer-induced pressure fluctuations at mach 6, J. Fluid Mech. 822 (2017) 5-30.

[58] R. H. Kraichnan, Pressure fluctuations in turbulent flow over a flat plate, J. Acoust. Soc. Am. 28 (1956) 378-390.

[59] O. M. Phillips, On the aerodynamic surface sound from a plane turbulent boundary, Proc. R. Soc. A 234 (1956) $327-335$.

[60] R. L. Panton, J. H. Linebarger, Wall pressure spectra calculations for equilibrium boundary layers, J. Fluid Mech. 65 (1974) $261-287$.

[61] G. K. Batchelor, Pressure fluctuations in isotropic turbulence, Proc. Camb. Phil. Soc. 47 (1951) 359-374.

[62] G. Schewe, On the Structure and Resolution of Wall-Pressure Fluctuations Associated with Turbulent Boundary Layer Flow, J. Fluid Mech. 134 (1983) 311-328.

[63] Y. Tsuji, T. Ishihara, Similarity scaling of pressure fluctuation in turbulence, Phys. Rev. E 68 (2003) 026309.

[64] R. Camussi, M. felli, F. Pereira, G. Aloisio, A. D. Marco, Statistical properties of wall pressure fluctuations over a forward-facing step, Phys. Fluids 20 (2008) 075113.

[65] J. Laufer, Some statistical properties of the pressure field radiated by a turbulent boundary layer, Phys. Fluids 7 (1964) $1191-1197$. 